\title{
A self-extubation case series in an ICU after the introduction of an early mobilization project
}

\author{
Jim Tseng BS, Mark Sigler MD, Hawa Edriss MD, Alisha Turner MSN, \\ Kristi Valdez MSN, Kavitha Selvan BS, Kenneth Nugent MD
}

\begin{abstract}
Background: Recent studies demonstrate that early mobilization of patients with acute respiratory failure reduces ICU and hospital length of stay. This patient care activity necessarily requires coordinated efforts by ICU personnel and alert patients and has the potential for adverse outcomes, including unplanned extubation.

Methods: Our intensive care unit introduced an early mobilization quality improvement project in April 2014. This project involved an eight step program which was started as soon as the patient was medically stable. The nurse managers kept a log of patients who participated in this project and a log of all patients who self-extubated during this period.

Results: Twenty-five patients self-extubated during this time period; the event rate was 1.1 episodes per week in a 31 bed ICU. The mean age was $46.8 \pm 13.6$ years; $64 \%$ were men. The initial indications for mechanical ventilation in these patients included respiratory disease (40\%), sepsis (4\%), encephalopathy (8\%), and miscellaneous diagnoses (48\%). Initial chest x-ray readings included clear lung fields, infiltrates, effusions, and other abnormalities. Twelve episodes occurred on the day shift, and 13 episodes occurred on the night shift. The most recent Glasgow Coma Scale score in these patients was 11.8 (mean) with a range of 8-15. Eighty percent of the patients were restrained, $40 \%$ were on analgesics, and $56 \%$ were on sedatives. The mean $\mathrm{FiO}_{2}$ at the time of self-extubation was $57.3 \pm 29 \%$, and the mean PEEP level was $5.4 \pm 1.5 \mathrm{~cm} \mathrm{H}_{2} \mathrm{O}$. Seven patients (28\%) required reintubation. None of these patients in the early mobilization project had an episode of self-extubation.

Conclusions: The patients who self-extubated in our ICU had no unique characteristics which might help us identify them before these events occurred. This did not occur in the patients in the early mobilization project. Self-extubation events provide a good monitor for ICU care. In our ICU the frequency of reintubation was low, and this might suggest that we need to manage our weaning protocols better with earlier extubation in some patients.
\end{abstract}

Key words: self-extubation, mechanical ventilation, sedation.

Corresponding author: Kenneth Nugent, MD

Contact Information: Kenneth.nugent@ttuhsc.edu. DOI: 10.12746/swrccc2014.0208.098 


\section{INTRODUCTION}

Many patients with acute respiratory failure require mechanical ventilation with positive airway pressures to deliver tidal volumes. These patients usually have an endotracheal tube to secure their airways and to provide a seal in the airway so that the positive pressure ventilation is effective. These endotracheal tubes are uncomfortable because they prevent speech, inhibit routine swallowing, and may cause acute airway injury resulting in either bronchospasm or pain. In addition, this situation is frightening and stressful, and most patients receive both narcotics to reduce pain and sedation to reduce anxiety. Patient management in the ICU requires continuous attention to comfort and sedation. Over-sedation is undesirable because it usually prolongs the ICU stay and the time on a mechanical ventilator. ${ }^{1}$ Under-sedation is undesirable because patients are uncomfortable and can interfere with their own care. We recently introduced an early mobilization program in our ICU for patients on mechanical ventilators in an effort to limit the loss of muscle function in these patients and hopefully expedite weaning and extubation. ${ }^{2,3}$ This program necessarily requires an alert and cooperative patient and possibly the use of less sedation. We were concerned about adverse events, including unplanned extubation, during this project. Consequently, we kept a log of patients who participated in the early mobilization project with particular attention to adverse events and a log of all patients who self-extubated during this time period for comparisons if unplanned extubations occurred.

\section{Methods}

The medical intensive care unit at University Medical Center in Lubbock, TX, introduced an early mobilization project for patients on mechanical ventilators on April 1, 2014. This project used an eight step ladder of increasing physical activity in patients on ventilators. The second step required some active muscular effort by the patient. The last two steps involved sitting in the chair and ambulating with assistance. This project required a multidisciplinary effort involving physicians, nurses, respiratory therapists, and physical therapists and increased attention to patient comfort, alertness, and safety. Analgesia was initially addressed with narcotic administration (usually fentanyl), and sedatives were added if analgesics did not provide an adequate comfort level. In the event that sedation was required propofol or dexmedetomidine were used in accordance with the Society for Critical Care Medicine 2013 guidelines. Benzodiazepines were used in patients who did not respond to the preferred drugs or had clear contraindications. Reducing the use of sedation allows more patient participation in the mobilization activities but also increases the possibility of more anxiety and interference with routine care. One important concern was whether or not there would be an increase in the number of unplanned extubations. The nurse manager kept a log of all patients who self-extubated during the period from April 1 through September 2, 2014. These charts were retrospectively reviewed to determine patient demographics, characteristics of acute respiratory failure, management strategies around the time of extubation, and outcomes. This study was approved by the Institutional Review Board at Texas Tech University Health Sciences Center in Lubbock.

\section{RESULTS}

Twenty-five patients self-extubated between April 1, 2014, and September 2, 2014; the overall rate was 1.1 episodes per week in a 31 bed medical ICU. The mean age was $46.8 \pm 13.6$ years, and $64 \%$ were men. The mean BMI was $29.2 \pm 8.6 \mathrm{~kg} / \mathrm{m}^{2}$. Initial indications for mechanical ventilation included respiratory disease $(40 \%)$, sepsis $(4 \%)$, encephalopathy $(8 \%)$, and other miscellaneous diagnoses (48\%). Initial chest $\mathrm{x}$-ray readings included clear lung fields $(36 \%)$, infiltrates $(40 \%)$, effusions (4\%), and other abnormalities (20\%).

This unit consistently has a one nurse to two patient ratio on all shifts. Five patients $(20 \%)$ who self-extubated were in "corner" rooms. Twelve episodes occurred between 7AM and 7 PM, and 13 occurred between 7PM and 7 AM. Three episodes occurred between $6 \mathrm{AM}$ and $8 \mathrm{AM}$ and $6 \mathrm{PM}$ and $8 \mathrm{PM}$ during shift changes. The most recent Glasgow coma scale scores averaged 11.8 with a range of 8-15 (allowing writing during intubation to count as a verbal 5 
score). Eighty percent of the patients were restrained, $40 \%$ were receiving analgesics (fentanyl), and $56 \%$ were receiving sedatives (propofol, or dexmedetomidine, or benzodiazepines) at the time of self-extubation. Weaning was an active consideration in $64 \%$ of the patients. The mean $\mathrm{FiO}_{2}$ was $57.3 \pm 29 \%$ (range: 30-100\%); the mean PEEP level was $5.4 \pm 1.5 \mathrm{~cm}$ $\mathrm{H}_{2} \mathrm{O}$ (range: $5-12 \mathrm{~cm} \mathrm{H}_{2} \mathrm{O}$ ). Seven patients (28\%) required reintubation. The mean $\mathrm{PaO}_{2} / \mathrm{FiO}_{2}$ ratios were $237.2 \pm 118.8$ in patients not requiring reintubation and $227.2 \pm 99.0$ in patients requiring reintubation $(P$ $>0.05$ ). No patient on the early mobilization protocol self-extubated.

\section{Discussion}

Self-extubation occurs relatively infrequently in our medical intensive care unit. The event rate is approximately one per week for all patients in our 31 bed unit. The patient group included both men and women with a wide range of ages and underlying medical conditions requiring mechanical ventilation. Most patients had a relatively high Glasgow coma scale score. The Richmond agitation sedation scale score was not recorded frequently enough near the time of extubation to provide useful information about the level of agitation in these patients. However, most patients $(80 \%)$ were restrained, and most (more than $50 \%$ ) were receiving either analgesia or sedation or both at the time of self-extubation. The outcomes were relatively good, and only seven patients $(28 \%)$ required reintubation.

Factors relevant to self-extubation include management strategies, such as the use of restraints, sedation, and the nurse to patient ratio. ${ }^{4,5}$ Balon reported that $80 \%$ of the patients in her study were restrained but less than one third had either an analgesic or sedative medication given intravenously within four hours of extubation. ${ }^{6}$ She suggested that "as needed dosing" contributed to these events and that continuous infusions might be preferable. Tung, et al. found that patients with self-extubation had received benzodiazepines and considered the possibility that paradoxic reactions to these drugs led to these outcomes. ${ }^{7}$ Moons developed a risk assessment tool based on the Bloomsbury sedation score in the Glasgow Coma Scale. ${ }^{8}$ Patients at increased risk for self-extubation had low sedation levels and higher levels of consciousness. Singh reported self-extubations in a medical-surgical unit which consistently had a one to one nurse to patient ratio and targeted sedation scores characterized as "tranquil and obeying commands to asleep". ${ }^{9}$ Consequently, a high nurse to patient ratio does not prevent self-extubation. Patient related factors include gender (more frequent in males in some studies), agitation, and potentially the duration of intubation. Physician related factors include inadequate attention to analgesia and sedation and slow decision making regarding the potential for extubation. The frequency of reintubation ranged from $25 \%$ in our study to $94 \%$ in the Singh study. ${ }^{9}$

Our study did not identify any typical characteristics of patients who self-extubate. Therefore, there is no obvious method to focus patient care and preventive strategies in patients who are at higher risk for self-extubation. However, tracking the frequency of self-extubation provides important quality of care information in all ICUs which use mechanical ventilation for respiratory support. This group of patients is a relatively small percentage of the total number of patients who receive ICU care. However, medical and nursing care strategies in these patients are likely relevant to all patients in ICUs. In situations in which patients self-extubate and require reintubation, it seems clear that these patients need more attention to sedation and possibly the use of restraints. Both the level of alertness and the level of agitation influence the frequency of self-extubation, and this information is relatively easily recorded at the bedside. This, in turn, would influence the use of analgesia and sedation. Situations in which patients self-extubate but do not require reintubation suggest that the management of weaning and extubation needs more attention. In addition, the outcomes in patients who self-extubate and then require reintubation are clearly worse than in patients who do not require reintubation. These patients have longer lengths of stay, more frequent ventilator-associated pneumonia, and increased mortality rates and need careful review of their diagnoses and comorbidities at the time of reintubation. ${ }^{10}$ 


\section{RECOMMENDATIONS}

1. Intensive care units with intubated patients need to use a consistent sedation scoring method to track the level of patient sedation and patient comfort. However, nurses and physicians need to remember the sedation levels may not be stable in some patients. For example, patients recovering from drug overdoses or from seizures may have rapid changes in their levels of consciousness as their medical problems resolve. In addition, some patients may have unpredictable responses to analgesia and sedation. This group likely includes patients on chronic narcotics and on chronic benzodiazepines.

2. Intensive care units need to use an orderly and organized process for weaning and extubation. This activity should be a priority each morning since avoidable delays likely increase the frequency of selfextubation as the patient's analgesia and sedation wear off and the level of consciousness increases.

3. Patients who self-extubate and then require reintubation represent a special subgroup with poor outcomes. These patients need careful reassessment at the time of reintubation to identify any treatable factors resulting in respiratory failure.

Author Affiliation: Jim Tseng and Kavitha Selvan are students in the School of Medicine. Hawa Edriss is a resident in Internal Medicine; Mark Sigler is a fellow in pulmonary and critical medicine. Alisha Turner and Kristi Valdez are nurses at University Medical Center in Lubbock, TX. Kenneth Nugent is in the pulmonary and critical care division at TTUHSC.

Received: 09/25/2014

Accepted: 10/05/2014

Reviewers: Cynthia Jumper MD

Published electronically: 10/15/2014

Conflict of Interest Disclosures: None

\section{REFERENCES}

1. Kress JP, Pohlman AS, O'Connor MF, et al. Daily interruptions of sedative infusions in critically ill patients undergoing mechanical ventilation. New Engl J Med 2000; 342:1471-7.

2. Bailey P, Thomsen GE, Spuhler VJ, et al. Early activity is feasible and safe in respiratory failure patients. Crit Care Med 2007; 35(1):139-145.

3. Morris PE, Goad A, Thompson C, et al. Early intensive care unit mobility therapy in the treatment of acute respiratory failure. Crit Care Med 2008; 36(8): 2238-2244.

4. de Groot RI, Dekkers OM, Herold I, de Jonge E, Arbous M. Risk factors and outcomes after unplanned extubations on the ICU: a case-control study. Critical Care 2011; 15:R19.

5. Chang LC, Liu PF, Huang YL, Yang SS, Chang WY. Risk factors associated with unplanned endotracheal self-extubation of hospitalized intubated patients: a 3-year retrospective case-control study. Appl Nurs Res 2011 Aug; 24(3):188-192.

6. Balon JA. Common factors of spontaneous selfextubation in a critical care setting. Int J Trauma Nurs 2001 Jul-Sept; 7(3):93-99.

7. Tung A, Tadimeti L, Caruana-Montaldo B, Atkins PM, Mion LC, Palmer RM, Slomka J, Mendelson W. The relationship of sedation to deliberate self-extubation. J Clin Anesth 2001 Feb; 13(1):24-29.

8. Moons P, Sels K, De Becker W, De Geest S, Ferdinande P. Development of risk assessment tool for deliberate self-extubation in intensive care units. Intensive Care Med 2004 Jul; 30(7):1348-1355.

9. Singh PM, Rewari V, Chandralekha, Arora MK, Trikha A. A retrospective analysis of determinants of selfextubation in a tertiary care intensive care unit. J Emerg Trauma Shock 2013 Oct-Dec; 6(4):241-245.

10. Chen CM, Chan KS, Fong Y, Hsing SC, Cheng AC, Sung MY, Su MY, Cheng KC. Age is an Important Predictor of Failed Unplanned Extubation. International J Gerontology 2010 September; 4(3):120-129. 